\title{
Tratamento cirúrgico da embolia pulmonar crônica: análise da experiência inicial
}

\author{
Fábio B. JATENE*, Paulo M. PÊGO-FERNANDES*, Sérgio POVEDA*, Rosângela MONTEIRO*, \\ Alberto CUKIER ${ }^{\star}$, Charles MADY* ${ }^{\star}$ Adib D. JATENE*
}

JATENE, F. B.; PÊGO-FERNANDES, P. M.; POVEDA, S.; MONTEIRO, R.; CUKIER, A.; MADY, C.; JATENE, A. D. - Tratamento cirúrgico da embolia pulmonar crônica: análise da experiência inicial. Rev. Bras. Cir. Cardiovasc, $10(2): 70-76,1995$.

RESUMO: Após a tromboembolia pulmonar aguda, na maioria dos casos, ocorre lise com recanalização dos ramos pulmonares. Entretanto, em pequeno, mas indeterminado número de pacientes (pts) com embolia pulmonar (EP) crônica, a incompleta resolução do material embólico pode resultar em grave hipertensão pulmonar. A tromboendarterectomia pulmonar (TP) é uma opção, especialmente quando a obstrução é proximal e os pts apresentam falência ao tratamento clínico. Dentre as técnicas cirúrgicas já empregadas, a que utiliza, atualmente, a esternotomia com circulação extracorpórea (CEC) e parada circulatória total (PCT) é a que tem apresentado melhores resultados. Até janeiro/95 foram operados 15 pts portadores de EP recurrente. A arteriografia pulmonar mostrou comprometimento bilateral em $66,7 \%$ dos casos. Esternotomia foi realizada em $73,3 \%$ dos pts. Nestes pts, a técnica utilizada foi a TP por traçăo retrógrada dos trombos. Circulação extracorpórea foi utilizada em todos os pts, com tempo médio de $124,8 \mathrm{~min}$, sendo em $66,7 \%$ dos casos realizada PCT, com tempo médio de $34,2 \mathrm{~min}$. Os valores pressóricos médios na artéria pulmonar eram $91 / 32 / 55 \mathrm{mmHg}$ no pré e apresentaram queda para $52 / 15 / 27 \mathrm{mmHg}$ no pós-operatório. Houve $1(6,7 \%)$ óbito hospitalar por coagulopatia e 1 tardio devido a problemas não relacionados à operação. Dos 13 pts vivos, 1 $(7,7 \%)$ apresentou reobstrução e $12(92,3 \%)$ encontram-se assintomáticos sob anticoagulação oral, tendo retornado às atividades habituais, em um período de seguimento de até 165 meses (média $47 \mathrm{~m}$ ). Em conclusão, a TP realizada por esternotomia e, quando necessário, com auxílio de PCT é procedimento viável e seguro para resoluçāo de EP crônica, levando à remissão da sintomatologia destes pts e à manutenção do resultado a longo prazo.

DESCRITORES: Embolia pulmonar, cirurgia. Tromboendarterectomia pulmonar. Hipertensão pulmonar.

\section{INTRODUÇÃO}

Após a tromboembolia pulmonar aguda, na maioria dos casos, ocorre, em determinado período, recanalização dos ramos pulmonares 9,10 . Entretanto, em um número pequeno mas indeterminado de pacientes (pts), a incompleta lise do material embólico, seguida de sua organização pode resultar em severa hipertensão pulmonar, caracterizando a embolia pulmonar (EP) crônica 1, 11-14. Dos cerca de 500000 novos casos estimados por ano de EP, nos EUA, 5 entre 2500 e 18000 poderiam evoluir desta maneira, segundo CHITWOOD et alii ${ }^{4}$.

Nesta situação, o tratamento clínico com anticoagulantes ou trombolíticos tem pequena ou nenhuma ação e o tratamento cirúrgico praticamente se impõe.

Introduzida há vários anos, a retirada cirúrgica dos êmbolos não apresentou, inicialmente, resultados consistentes 3,25 . Com relação ao ato opera-

Trabalho realizado no Instituto do Coração do Hospital das Clínicas da Faculdade de Medicina da Universidade de São Paulo. Apresentado ao $22^{\circ}$ Congresso Nacional de Cirurgia Cardíaca. Brasília, DF, 30 de março a $1^{2}$ de abril, 1995.

* Do Instituto do Coração do Hospital das Clínicas da Faculdade de Medicina da Universidade de São Paulo.

Endereço para correspondência: Fábio B. Jatene. Av. Dr. Enéas de Carvalho Aguiar, 44. Divisão Cirúrgica. CEP 05403-000 São Paulo, SP, Brasil. 
JATENE, F. B.; PÊGO-FERNANDES, P. M.; POVEDA, S.; MONTEIRO, R.; CUKIER, A.; MADY, C.; JATENE, A. D. - Tratamento cirúrgico da embolia pulmonar crônica: análise da experiência inicial. Rev. Bras. Cir. Cardiovasc., 10 (2): 70-76, 1995.

tório, havia muita controvérsia quanto a via de acesso (toracotomia lateral ou esternotomia), a utilidade da circulação extracorpórea (CEC), a abordagem uni ou bilateral etc. DAILY et alii ${ }^{5-8} \mathrm{e}$, posteriormente, JAMIESON et alii ${ }^{12}$ e MOSER et alii ${ }^{15-19}$, do mesmo centro em San Diego, Califórnia, tiveram um importante papel no desenvolvimento e sistematização desta operação. Dentre todas as técnicas empregadas, a que utiliza, atualmente, esternotomia, CEC e parada circulatória total (PCT) é a que tem apresentado melhores resultados, com morbimortalidade progressivamente menor 12 .

O objetivo deste trabalho é a avaliação de um grupo de pts portadores desta afecção, operados para ressecção dos trombos, visando analisar a técnica operatória em função dos resultados imediatos, bem como a evolução tardia.

\section{CASUISTICA E MÉTODOS}

Até janeiro de 95 foram operados 15 pts portadores de EP crônica. Dez $(66,7 \%)$ eram do sexo masculino e a idade variou de 22 a $74(41,8 \pm 13,3)$ anos.

Em todos os pts havia ocorrido trombose venosa profunda dos membros inferiores e em 1 havia também plaquetose associada. Quatoze $(93,3 \%)$ pts apresentavam dispnéia a mínimos esforços e ascite estava presente em $2(13,3 \%)$ pacientes.

Vários exames complementares foram realizados, como cintilografia pulmonar, mapeamento cardíaco, tomografia computadorizada e ressonância nuclear magnética. Entretanto, o diagnóstico definitivo e anatômico foi firmado por arteriografia pulmonar, realizada em todos os pacientes.

Havia hipertensão pulmonar em $14(93,3 \%)$ pts e os valores médios observados na artéria pulmonar foram 91/32/55 $\mathrm{mmHg}$.

Havia ligadura prévia da veia cava inferior em $1(6,7 \%)$ pt e da veia femoral direita em outro $(6,7 \%)$. Em $2(13,3 \%)$ pts a veia cava inferior estava obstruída e em $1(6,7 \%)$ havia sido colocado dispositivo tipo "umbrella".

Com relação ao comprometimento, em $66,7 \%$ dos casos ele era bilateral. Acometimento simultâneo dos lobos inferior e médio direitos e inferior esquerdo, ocorreu em $5(33,3 \%)$; pulmão direito 4 $(26,6 \%)$; pulmão esquerdo, lobos superior e médio direitos $3(20 \%)$; lobos superior e inferior à esquerda $1(6,7 \%)$ pulmão direito e lobo inferior esquerdo $1(6,7 \%)$ e lobos superior e inferior esquerdo e superior $e$ inferior direito em $1(6,7 \%)$ pt. (Tabela 1 e Figura 1).
TABELA 1

LOCAIS DE ACOMETIMENTO ARTERIAL PULMONAR

\begin{tabular}{lcr}
\hline & $N^{2}$ DE PACIENTES & $\%$ \\
\hline LID, LM, LIE & 5 & 33,3 \\
Pumão D & 4 & 26,6 \\
Pulmão E, LSD, LM & 3 & 20,0 \\
LSE, LIE & 1 & 6,7 \\
Pulmão D, LIE & 1 & 6,7 \\
LSE, LIE, LSD, LID & 1 & 6,7 \\
\hline Total & 15 & 100,0 \\
\hline
\end{tabular}

$\mathrm{L}=$ lobo; $\mathrm{I}=$ inferior; $\mathrm{S}=$ superior; $\mathrm{M}=$ médio; $\mathrm{D}=$ direito; $\mathrm{E}=$ esquerdo.

Quanto à via de acesso, a esternotomia foi utilizada em $11(73,3 \%)$ pts, a bitoracotomia anterior em $3(20 \%)$ e a toracotomia esquerda com ampliação à direita e secção transversal do esterno em $1(6,7 \%)$.

A CEC foi utilizada em todos os pts, sendo em $5(33,3 \%)$ com hipotermia moderada e em $10(66,7 \%)$ com hipotermia profunda e PCT. O tempo médio de CEC foi de $124,8 \pm 37,7 \mathrm{~min}$ e o tempo de PCT variou de 3 a $55(34,2 \pm 20,6)$ minutos.

\section{TÉCNICA OPERATÓRIA}

Nos primeiros 4 pts a técnica operatória sofreu modificações de caso a caso, procurando a obtenção de melhores resultados. Foi utilizada bitoracotomia anterior em 4 pts, pois se considerava importante, além da abordagem central da artéria pulmonar, o acesso pleural e periférico do pulmão.

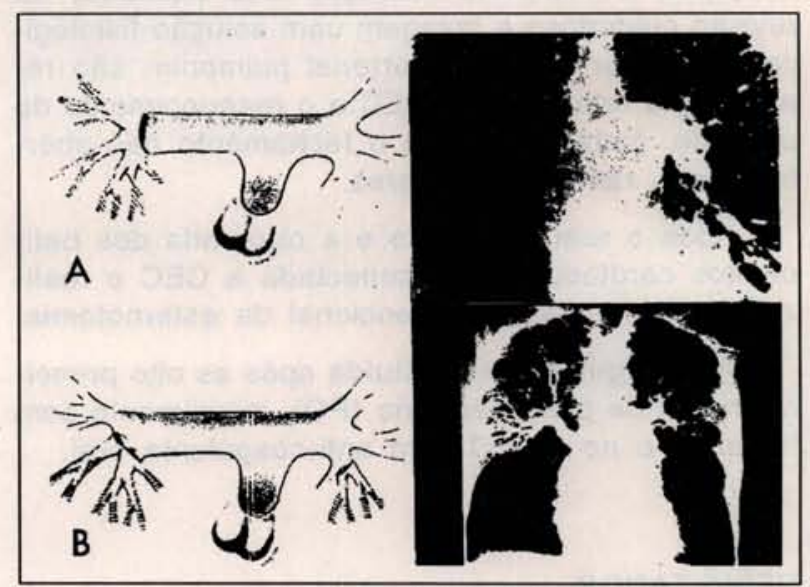

Fig. 1 - Esquema dos trombos no interior da árvore arterial pulmonar com a correspondente arteriografia em 2 pacientes. A: obstrução de toda a artéria pulmonar direita; B: obstrução dos ramos para os lobos médio e inferior à direita e inferior à esquerda. 
JATENE, F. B.; PÊGO-FERNANDES, P. M.; POVEDA, S.; MONTEIRO, R.; CUKIER, A.; MADY, C.; JATENE, A. D. - Tratamento cirúrgico da embolia pulmonar crônica: análise da experiência inicial. Rev. Bras. Cir. Cardiovasc., 10 (2): 70-76, 1995.

Somente nos 2 primeiros pts foi ampliada a artéria pulmonar, na cisura, o que foi abandonado a seguir. A técnica atualmente empregada e que foi utilizada nos últimos 11 pts é a que tem se revelado mais adequada e cuja descrição é a que segue. Compreende a realização de esternotomia longitudinal mediana e instalação de CEC com drenagem venosa por duas cânulas em veias cava superior $e$ inferior e retorno por cânula arterial em aorta ascendente. Durante o resfriamento entre $15^{\circ} \mathrm{C}$ e $20^{\circ} \mathrm{C}$ é feita ampla dissecção entre a aorta ascendente e o tronco e ramos pulmonares direito e esquerdo. É realizada cuidadosa inspeção do átrio direito, que pode apresentar trombos no seu interior. Após instalação da PCT, é aberta inicialmente a artéria pulmonar direita, entre a aorta e a veia cava superior, e o cirurgião colocado à esquerda do pt realiza a retirada dos trombos presentes nos ramos da artéria pulmonar direita. Esta ressecção se faz por tração retrógrada dos trombos, no plano da endartéria pulmonar, caracterizando uma tromboendarterectomia, muito facilitada com auxílio de pequeno aspirador (Figura 2). Após liberação do trombo até ramos lobares segmentares e subsegmentares, é feita a sua retirada, que se caracteriza como um molde do interior da árvore arterial pulmonar direita (Figura 3). Após estas manobras, a circulação é momentaneamente retomada, enquanto se procede ao preparo para a abordagem dos ramos pulmonares à esquerda. Após cerca de 10 a 15 minutos de CEC, com a temperatura mantida, é aberto o tronco pulmonar em direção à artéria pulmonar esquerda. $O$ cirurgião colocado do lado oposto ou à direita do pt realiza as mesmas manobras já descritas previamente com a ressecção do trombo, no plano da endartéria pulmonar.

Após a completa remoção dos trombos, da revisão cuidadosa e lavagem com solução fisiológica do interior da árvore arterial pulmonar, são realizadas a retomada da CEC e o reaquecimento do paciente, juntamente com o fechamento das aberturas nos ramos pulmonares.

Após o reaquecimento e a retomada dos batimentos cardíacos, é desconectada a CEC e realizado o fechamento convencional da esternotomia.

Anticoagulação é instituída após as oito primeiras horas de pós-operatório (PO), inicialmente com heparina e no $3^{\circ} \mathrm{PO} \mathrm{com}$ anti-coagulante oral.

\section{RESULTADOS}

Em todos os pts obteve-se a retirada dos trombos do interior da árvore arterial pulmonar. Em $2(13,3 \%)$ pts foram retirados, também, trombos do

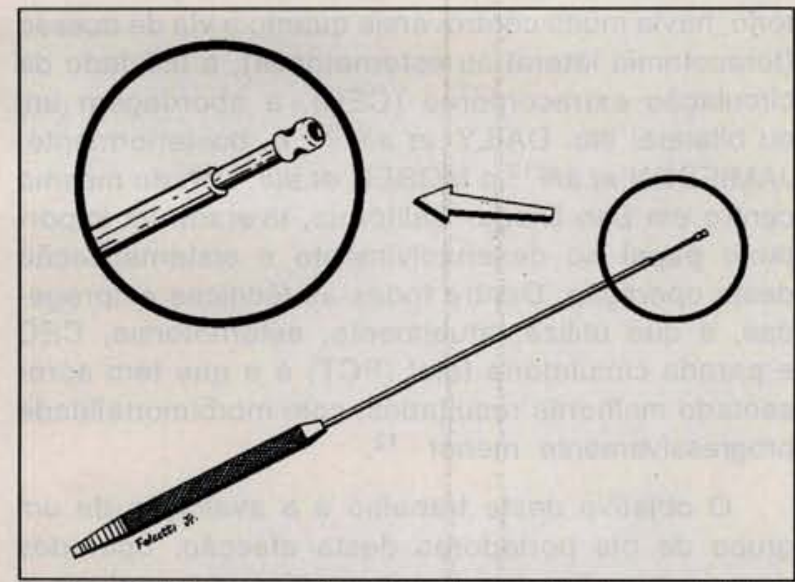

Fig. 2 - Detalhe esquemático do aspirador utilizado para facilitar a dissecção do trombo juntamente com a endarterectomia.

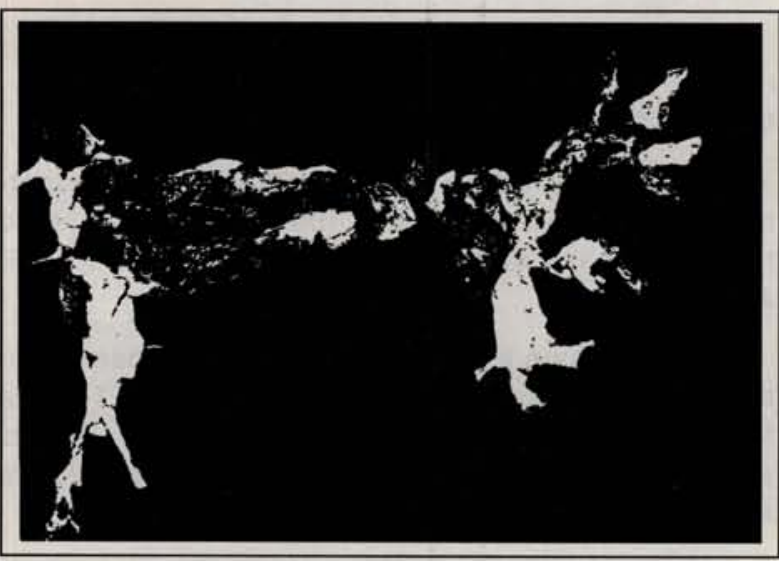

Fig. 3 - Foto do espécime retirado do interior da árvore arterial de um paciente. A semelhança de um molde, apresenta trombos e a endartéria com as ramificações dos ramos lobares, segmentares e subsegmentares.

interior do átrio direito. O fluxo retrógrado após a retirada dos trombos esteve presente em 14 (93,3\%) dos pacientes.

Houve queda da pressão arterial pulmonar em $10(66,7 \%)$ pts. Os valores obtidos após a operação foram $52 / 15 / 27 \mathrm{mmHg}$, com queda de $57 \%$ em relação aos valores pré-operatórios. A situação da veia cava inferior após a operação pode ser avaliada na Tabela 2. Em $8(53,3 \%)$ dos casos ela foi

TABELA 2

SITUAÇÃO DA VEIA CAVA INFERIOR

\begin{tabular}{lccc}
\hline & PRÉ-OP & PÓS-OP & TOTAL \\
\hline Umbrella & $1(6,7 \%)$ & - & $5(33,3 \%)$ \\
Obstruída & $2(13,3 \%)$ & $3(20 \%)$ & $2(13,3 \%)$ \\
Ligadura & $2^{*}(13,3 \%)$ & - & $1(6,7 \%)$ \\
\hline
\end{tabular}

* 1 caso; femoral direita

$\mathrm{OP}=$ operatório 
JATENE, F. B.; PÊGO-FERNANDES, P. M.; POVEDA, S.; MONTEIRO, R.; CUKIER, A.; MADY, C.; JATENE, A. D. - Tratamento cirúrgico da embolia pulmonar crônica: análise da experiência inicial. Rev. Bras. Cir. Cardiovasc., 10 (2): 70-76, 1995.

submetida a alguma forma de intervenção do seu fluxo.

Houve $1(6,7 \%)$ óbito hospitalar por coagulopatia no $3^{\circ}$ dia de pós-operatório. Houve $1(6,7 \%)$ óbito tardio devido a problemas não relacionados à operação. A alta hospitalar dos 14 pts que sobreviveram ocorreu entre 10 e 26 (16) dias. Dos 13 pts vivos, $1(7,7 \%)$ apresentou reobstrução e $12(92,3 \%)$ encontram-se assintomáticos, com bom resultado funcional sob anticoagulação oral, tendo retornado às atividades habituais, em período de seguimento de até 165 meses (média $47 \mathrm{~m}$ ). Estes dados podem ser avaliados no Gráfico 1 .

\section{COMENTÁRIOS}

A ocorrência da tromboembolia pulmonar, além de freqüente, pode apresentar características muito diversas; pode variar desde episódios sem maior repercussão clínica, até processos extremamente severos levando a oclusão dos maiores ramos arteriais pulmonares, com todas as graves repercussões decorrentes 20,21. Além disto, pode se manifestar como episódio isolado ou como episódios sucessivos. Na maioria dos casos a resolução dos êmbolos provoca a recanalização dos ramos pulmonares $e$ isto pode ocorrer espontânea ou medicamentosamente ${ }^{10}$. Para os quadros agudos sem maiores repercussões clínicas, o tratamento medicamentoso, especialmente com o uso de heparina, é largamente aceito. Entretanto, os episódios embólicos agudos severos, com risco de vida imediato são, já há vários anos, abordados na literatura como de tratamento controverso $14,24,27,28$, 30. A utilização de trombolíticos para a lise ou da cirurgia para retirada dos trombos agudos vem sendo advogada, sem definição exata do melhor método. Nos dias atuais, esta controvérsia ainda persiste,

GRÁFICO 1

EMBOLIA PULMONAR CRÓNICA SOBREVIDA ATUARIAL PÓS-EMBOLECTOMIA

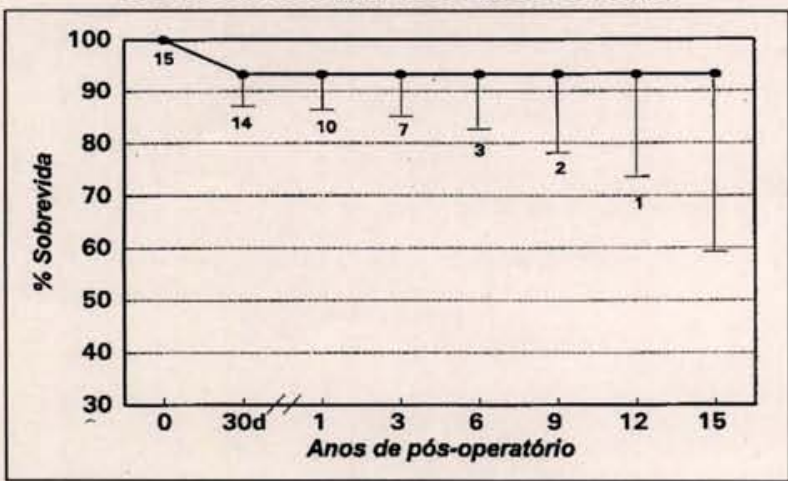

havendo maior tendência atual do uso de trombolíticos, ressaltando-se, aí, a análise de caso a caso ${ }^{2}$.

Com relação ao tratamento dos episódios tromboembólicos chamados crônicos, nos quais não houve lise dos trombos, posicionados no interior da árvore arterial pulmonar por processos embólicos sucessivos, é fácil perceber que a ação medicamentosa praticamente não tem ação $22,23,26$. Assim sendo, a abordagem cirúrgica naturalmente torna-se o método de escolha. Entretanto, embora possa parecer lógica tal abordagem, tecnicamente, ao longo dos anos, apresentou uma evolução difícil e dúbia. Inicialmente, foi preconizada a abordagem do lado mais comprometido e a cirurgia realizada por toracotomia póstero-lateral, sem $\mathrm{CEC}^{3}$. Se, por um lado, este procedimento seria de fácil acesso aos cirurgiões especializados e constituiria uma operação menos elaborada, por outro lado, trouxe uma série de problemas, especialmente dificuldades para uma perfeita retirada dos trombos 12,19 . Ao contrário dos processos agudos, os trombos organizados (de cor clara) estão firmemente aderidos ao endotélio dos ramos arteriais pulmonares e a sua retirada deve ser muito cuidadosa e detalhada. Atualmente, considera-se correto englobar a retirada da camada interna da artéria, caracterizando, portanto, o procedimento como uma tromboendarterectomia arterial pulmonar. Assim, ao longo dos anos, percebeu-se que a utilização da CEC facilitaria e tornaria mais completo e criterioso o procedimento. Outro ponto interessante seria a melhor via de acesso. Com auxílio da circulação extracorpórea, o acesso anterior traria inúmeras facilidades para a introdução das cânulas venosas e arterial. A incisão de escolha seria a esternotomia. Entretanto, com a esternotomia praticamente ficaria afastada a possibilidade de um bom acesso aos ramos mais distais da artéria pulmonar, especialmente nas regiões cisurais. Assim, alguns autores advogavam toracotomias unilaterais ou bilaterais anteriores, com seção transversal do esterno 8,25 . Nós estávamos entre os que utilizavam esta incisão no início da nossa experiência. Entretanto, apesar da ótima exposição, esta incisão é muito agressiva e ficamos convencidos de que não há a necessidade da abordagem da cisura ou do espaço pleural, nestes casos. A abordagem central do tronco pulmonar e das artérias pulmonares direita e esquerda promove perfeita visibilização dos trombos no interior dos ramos lobares segmentares e subsegmentares e, por descolamento e tração retrógrada, a sua completa retirada. A bitoracotomia era, no início, a nossa incisão preferencial. Entretanto, no paciente que evoluiu a óbito, atribuímos à incisão 0 intenso sangramento decorrente da lise das aderências entre as pleuras, que foram desfeitas sob 
JATENE, F. B.; PÊGO-FERNANDES, P. M.; POVEDA, S.; MONTEIRO, R.; CUKIER, A.; MADY, C.; JATENE, A. D. - Tratamento cirúrgico da embolia pulmonar crônica: análise da experiência inicial. Rev. Bras. Cir. Cardiovasc., 10 (2): 70-76, 1995.

o pretexto de ampla exposição da artéria na cisura, com severa coagulopatia e óbito. Desde então, a esternotomia tem sido a incisão preferencial para os nossos pacientes e com bons resultados. Ainda com relação à circulação extracorpórea e uso de PCT, alguns pontos devem ser comentados. Um dos aspectos que caracteriza o bom resultado da operação é a presença de fluxo retrógrado ou distal dos ramos pulmonares após a retirada dos trombos, é o chamado back-flow ou back-bleeding e caracteriza o bom prognóstico da operação. Entretanto, devido especialmente a esta característica, fica difícil, em alguns casos, mesmo sob CEC, realizar perfeita tromboendarterectomia. A PCT, facilita muito o procedimento, que pode ser feito com toda a tranqüilidade e rigor técnico. Na nossa experiência, a PCT foi usada sempre que necessário, ou seja, nos casos com sangramento retrógrado intenso e dificuldades à retirada correta dos trombos. Não tivemos complicações decorrentes da PCT, mesmo em indivíduos idosos, embora haja descrição de complicações neurológicas dela decorrentes ${ }^{31}$. Os valores médios descritos por JAMIESON et alii ${ }^{12}$ de PCT foram de $45 \mathrm{~min}$ e os nossos de $34,2 \mathrm{~min}$

Um outro ponto importante a ser comentado diz respeito ao tempo de evolução da doença e ao lado a ser operado. Pode ser difícil caracterizar um paciente quando abordado por primeira vez em relação ao tempo de evolução da doença e a sua indicação cirúrgica. Dispnéia intratável por métodos clínicos e evolução da doença por períodos superiores a 3 meses para alguns autores e a 6 meses para outros mantêm a indicação cirúrgica. Aceitase, atualmente, a abordagem bilateral, sempre que possível a via mais favorável. Alguns relatos da literatura, entre eles MOSER et alii ${ }^{15-19}$ confirmam - que temos observado. Os achados angiográficos são sempre menos exuberantes que os achados cirúrgicos e, com isto, há uma tendência a subvalorizar a extensão da doença, no pré-operatório 29. Além disto, considera-se que, para elevações de pressão na artéria pulmonar, o comprometimento deve ser sempre amplo, correspondendo a mais de um pulmão. Isto parece ter importante papel na evolução pós-operatória e a conseqüente queda da pressão no território arterial pulmonar. Tromboendarterectomias mais amplas tendem a reduzir mais a pressão em território pulmonar. A nossa experiência revela que, após a desobstrução, a evolução clínica foi muito positiva e, mesmo nos indivíduos nos quais não houve queda expressiva de pressão, a melhora clínica foi muito satisfatória. Obtivemos redução da pressão arterial pulmonar em $66,7 \%$ dos doentes de valores de $91 / 32 / 55$ para $52 / 15 / 27$, com melhora clínica em todos, em períodos médios de 47 meses e máximos de até 165 meses.

Atualmente, a cirurgia para correção da EPC é procedimento seguro, viável e com baixo índice de morbimortalidade. A técnica cirúrgica atualmente empregada e que apresenta melhores resultados é realizada por esternotomia, com CEC e PCT. A evolução dos doentes mostrou manutenção dos resultados imediatos a longo prazo com melhora clínica acentuada. 
JATENE, F. B.; PÊGO-FERNANDES, P. M.; POVEDA, S.; MONTEIRO, R.; CUKIER, A.; MADY, C.; JATENE, A. D. - Tratamento cirúrgico da embolia pulmonar crônica: análise da experiência inicial. Rev. Bras. Cir. Cardiovasc., 10 (2): 70-76, 1995.

RBCCV 44205-261

JATENE, F.B.; PÊGO-FERNANDES,P.M.; POVEDA, S.; MONTEIRO, R.; CUKIER, A.; MADY, C.; JATENE,

A. D. - Surgical treatmentent of chronic pulmonary embolism: analisys of initial experience. Rev. Bras. Cir. Cardiovasc., 10 (2): 70-76, 1995.

ABSTRACT: After the acute pulmonary embolism, in the majority of the cases lysis occurs with recanalization of the pulmonary branches. However, in a small but undetermined number of patients (pts) with chronic pulmonary embolism (PE) the incomplete resolution of the embolic material may result in a severe pulmonary hypertension. The pulmonary thromboendarterectomy is an option, specially when the obstruction is proximal and the patient presents failure of the clinical treatment. Many surgical techniques have been used, but the utilized sternotomy, extracorporeal circulation and hypothermic circulatory arrest presented best results. Until January 95,15 pts with PE were operated upon. The pulmonary angiography showed pulmonary embolism at both lungs in $66.7 \%$ of the cases. Sternotomy was realized in $73.3 \%$. Endarterectomy by retrograde traction of thrombus was used in these pts. Extracorporeal circulation was utilized in all pts, with medium time of $124.8 \mathrm{~min}$. In $66.7 \%$ of the cases hypothermic circulatory arrest was realized with medium time of $34.2 \mathrm{~min}$. The pulmonary artery pressure was $91 / 32 / 55 \mathrm{mmHg}$ in the pre and presented reduction by $52 / 15 /$ $27 \mathrm{mmHg}$ in the postoperative period. One hospitalar death due to coagulopathy, and one later death due to complications not related to surgery were registered. Of the 13 survivors, $1(7.7 \%)$ presented reobstruction and $12(92.3 \%)$ had good evolution and now are free of symptoms, with oral anticoagulation in a period of until 165 months $(47 \mathrm{mo})$. In conclusion, the pulmonary thromboendarterectomy realized by sternotomy and with help of the hypothermic circulatory arrest is a safe procedure for resolution of pulmonary embolus and symptoms, and maintenance of the results at longterm follow-up.

DESCRIPTORS: Embolism, pulmonary, surgery. Thromboendarterectomy, pulmonary. Hypertension, pulmonary.

\section{REFERÊNCIAS BIBLIOGRÁFICAS}

1 BALL, K. P.; GOODWIN, J. F.; HARRISON, C. V. Massive thrombotic occlusion of the large pulmonary arteries. Circulation, 14: 766-783, 1956.

2 BARUZZI, A. C. A.; TERZI, R. G.; SARAIVA, F. K.; GOLDBAUM, M.; COELHO, O. R.; NOGUEIRA, E. - Estreptoquinase no tromboembolismo pulmonar grave. Arq. Bras. Cardiol., 61: 287-293, 1993.

3 CABROL, C.; CABROL, A.; ACAR, J.; GANDJBAKHCH, I.; GUIRAUDON, G.; LAUGHLIN, L.; MATTEI, M. F.; GODEAU, P. - Surgical correction of chronic postembolic obstructions of the pulmonary arteries. J. Thorac. Cardiovasc. Surg., 76: 620-628, 1978.

CHITWOOD, W. R.; SABISTON, D. C.; WECHSLER, A. S. - Surgical treatment of chronic unresolved pulmonary embolism: Clin. Chest Med.; 5: 507-536, 1984.

5 DAILY, P. O.; DEMBITSKY, W. P.; IVERSEN, S.; MOSER, K. M.; AUGER, W. - Current early results of pulmonary thromboendarterectomy for chronic pulmonary embolism. Eur. J. Cardiothorac. Surg., 4: $117-123,1990$.

6 DAILY, P. O.; DEMBITSKY, W. P.; IVERSEN, S.; MOSER, K. M.; AUGER, W. - Risk factors for pulmonary thromboendarterectomy. J. Thorac. Cardiovasc. Surg., 99: 670-678, 1990.
7 DAILY, P. O.; DEMBITSKY, W. P.; PETERSON, K. L.; MOSER, K. M. - Modifications of techniques and early results of pulmonary thromboendarterectomy for chronic pulmonary embolism. J. Thorac. Cardiovasc Surg., 93: 221-233, 1987.

DAILY, P. O.; JOHNSTON, G. G.; SIMMONS, C. J.; MOSER, K.; M. - Surgical management of chronic pulmonary embolism. J. Thorac. Cardiovasc. Surg., 79: 523-531, 1980.

9 DALEN, J. E.; BANAS, J. S.; BROOKS, H. L.; EVANS, G. L.; PARASKOS, J. A.; DEXTER, L. - Resolution rate of acute pulmonary embolism in man. N. Engl. J. Med., 280: 1194-1199, 1969.

10 DANTZKER, D. R. \& BOWER, J. S. - Partial reversibility of chronic pulmonary hypertension caused by pulmonary thromboembolic disease. Am. Rev. Respir. Dis., 124: 129-131, 1981.

11 HOLLISTER, L. E. \& CULL, V. L. - The syndrome of chronic thrombosis of the major pulmonary arteries. Am. J. Med., 312-320, 1956.

12 JAMIESON, S. W.; AUGER, W. R.; FEDULLO, P. F.; CHANNICK, R. N.; KRIETT, J. M.; TARAZI, R. Y.; MOSER, K. M. - Experience and results with 150 pulmonary thromboendarterectomy operations over a 29-month period. J. Thorac. Cardiovasc. Surg., 106: 116-127, 1993. 
JATENE, F. B.; PÊGO-FERNANDES, P. M.; POVEDA, S.; MONTEIRO, R.; CUKIER, A.; MADY, C.; JATENE, A. D. - Tratamento cirúrgico da embolia pulmonar crônica: análise da experiência inicial. Rev. Bras. Cir. Cardiovasc., 10 (2): 70-76, 1995.

KEATING, D. R.; BURKEY, J. N.; HELLERSTEIN, H. K.; FEIL, H. - Chronic massive thrombosis of pulmonary arteries. Am. J. Roentgenol., 69: 208$220,1953$.

MILLER, G. A. H.; HALL, R. J. C.; PANETH, M. Pulmonary embolectomy, heparin and streptokinase: their place in the treatment of acute massive pulmonary embolism. Am. Heart J., 93: 568-574, 1977.

MOSER, K. M.; AUGER, W. R.; FEDULLO, P. F.; JAMIESON, S. W. - Chronic thromboembolic pulmonary hypertension: clinical picture and surgical treatment. Eur. Respir. J., 5: 334-342, 1992.

MOSER, K. M. \& BLOOR, C. M. - Pulmonary vascular lesions occurring in patients with chronic major vessel thromboembolic pulmonary hypertension. Chest., 103: 685-692, 1993.

MOSER, K. M.; DAILY, P. O.; PETERSON, K. DEMBITSKY, W.; VAPNEK, J. M.; SHURE, D.; UTLEY, J.; ARCHIBALD, C. - Thromboendarterectomy for chronic, major-vessel thromboembolic pulmonary hypertension. Ann. Intern. Med., 107: 560-565, 1987.

MOSER, K. M.; RHODES, P. G.; HUFNAGEL, C. C. Chronic unilateral pulmonary-artery thrombosis. $N$. Engl. J. Med., 272: 1195-1199, 1965.

MOSER, K. M.; SPRAGG, R. G.; UTLEY, J.; DAILY, P. O. - Chronic thrombotic obstruction of major pulmonary arteries. Ann. Intern. Med., 99: 299-305, 1983.

PARASKOS, J. A.; ADELSTEIN, S. J.; SMITH, R. E.; RICKMAN, F. D.; GROSSMAN, W.; DEXTER, L.; DALEN, J. E. - Late prognosis of acute pulmonary embolism. N. Engl. J. Med., 289: 55-58, 1973.

PATEL, J. J.; CHANDRASEKARAN, K.; MANIET, A. R. ROSS, J. J.; WEISS, R. L.; GUIDOTTI, J. A. - Impact of the incidental diagnosis of clinically unsuspected central pulmonary artery thromboembolism in treatment of critically ill patients. Chest, 105: 986$990,1994$.
RIEDEL, M.; STANEK, V.; WIDIMSKY, J.; PREROVSKY, I. - Longterm follow-up of patients with pulmonary thromboembolism. Chest, 81: 151-157, 1982.

23 SCHEIN, C. J.; RIFKIN, H.; HURWITT, E. S.; LEBENDIGER, A. - The clinical and surgical aspects of chronic pulmonary artery thrombosis. A. M. A. Arch. Intern. Med., 101: 592-605, 1958.

SHERRY, S. - Thrombolytic therapy of acute pulmonary embolism. Drug Therapy, 144-152, May 1983.

SNYDER, W. A.; KENT, D. C.; BAISCH, B. F. Successful endarterectomy of chronically occluded pulmonary artery. J. Thorac. Cardiovasc. Surg., 45: 482-489, 1963.

26 STELMACH, R.; FORTE, V.; MAYO, S. V.; NETTO, S. M.; ASHIDANI, M.; CUKIER, A. - Tromboembolismo pulmonar crônico. J. Pneumol., 15: 53-56, 1989.

27 SUTTON, G. C.; HALL, R. J. C.; KERR, I. H. - Clinical course and late prognosis of treated subacute massive, acute minor, and chronic pulmonary thromboembolism. Br. Heart J., 39: 1135-1142, 1977.

28 SUTTON, G. C.; HONEY, M.; GIBSON, R. V. - Clinical diagnosis of acute massive pulmonary embolism. Lancet, 1: 271-273, 1969.

29 TARDIVON, A. A.; MUSSET, D.; MAITRE, S.; BRENOT, F.; DARTEVELLE, P.; SIMONNEAU, G.; LABRUNE, M. - Role of CT in chronic pulmonary embolism: comparison with pulmonary angiography. J. Comp. Assist. Tomogr., 17: 345-351, 1993.

30 UROKINASE PULMONARY EMBOLISM TRIAL STUDY GROUP - Urokinase streptokinase embolism trial. JAMA, 229: 1606-1613, 1974.

31 WRAGG, R. E.; DIMSDALE, J. E.; MOSER, K. M.; DAILY, P. O.; DEMBITSKY, W. P.; ARCHIBALD, C. - Operative predictors of delirium after pulmonary thromboendarterectomy. J. Thorac. Cardiovasc. Surg., 96: 524-529, 1988. 